Abstracts

\title{
The 65th Annual General Meeting of the Scottish Paediatric Society
}

held in Edinburgh, November 27th, 1987

President: Dr. W. M.Fyfe 


\section{Clinical presentations}

\section{A CASE OF PURULENT PERICARDITIS}

\section{R. Dickson, J. Syme}

We present the case of F.T. a child of 11 weeks ( 3 weeks corrected age) who was found to have purulent pericarditis. He was treated with antibiotics alone without surgical intervention, and made a full recovery. F,T. (who was one of twins born at 32 weeks gestation) had a history of diarrhoea and intermittent fevers for one week, with poor feeding, irritability, and increasing lethargy for 24 hours.

On admission: He was extremely unwell, with poor peripheral perfusion, distended "doughy" abdomen, tachycardia, and tachypnoea. Initial investigations included: normal CSF microscopy and sugars, total white ce11 count 40,700 (neutrophils $73 \%$, lymphocytes $14 \%$, monocytes 13\%). Platelet count 685,000 , sodium 127 mmol/1, potassium $5.5 \mathrm{mmol} / 1$, and urea $5.2 \mathrm{mmol} / 1$. Admission chest $X$-ray was normal. Chest X-ray 20 hours after admission showed cardiomegaly.

littrasound then revealed a pericardial effusion, with small peritoneal and pleural effusions. The infant had inappropriate secretion of A.D.H. (plasma osmolality $265 \mathrm{mmol} / \mathrm{kg}$, urine osmolality $706 \mathrm{mmol} / \mathrm{kg}$ ) and hypoalbuminaemia, Subsequent ul.trasounds, showed f'ibrinous strands in the pericardial effusion and blood cultures grew Strep. pneumonia. With careful clinical monitoring, and the use of sequential cardiac ultrasounds to monitor the resolution of the pericardial effusion, surgical drainage procedures were not necessary.

Purulent pericarditis is discussed, with reference to the li.terature.

Paediatric Department, Western General Hospital, Eeinburgh.

CHRONIC GRANULOMATOUS DISEASE

N. J. Shaw, O. B. Eden, J. A. Raekurn

A 10-year-old boy presented at 10 months of age with a Staphylococcal abscess of the rectus sheath. Investigation revealed a raised white cell count, ESR, and immunoglobulins $G, A$ and $M$. Neutrophil function tests revealed absent reduction of Nitroblue tetrazolium, cecreased activity of the Hexose Monophosphate Shunt and diminished chemiluminescence. Despite this, Staphylococcal and candidal killing assays were within normal limits albeit at the lower end of the range. The child remained relatively well until the age of 10 when he developed a Staphylococcal hepatic abscess and a few months later sufferec an episode of salmonella septicaemia following a holicay in spain. The majority of our patient's tests of neutrophil function indicate that he has chronic granulomatous disease (CGD) although the normal Staphylococcal and candidal killing assays, together with the long intervals between severe infections, suggest that he may have a variant of this condition.

His brother has had recurrent infections and has similar abnormalities on neutrophil. function testing. The mother is well but has half normal values for these tests indicating that she is a carrier for CGD and therefore the mode of inheritance is $x-l i n k e d$ recessive.

Royal Hospital for Sick Children, Edinburgh.

ONDINE'S CURSE OR CONGENITAL CENTRAL HYPOVENTILATION SYNDROME ASSOCIATED WITH PSEUDOHIRSCHSPRUNG'S DISEASE A CASE REPORT

T. G. Marshall, A. T. Edmonds

A male infant weighing $2.32 \mathrm{~kg}$ at 37 weeks gestation was delivered by Haig-Ferguson forceps for foetal distress late in the second stage of labour. No resuscitation was required. During the first few hours of life, 2 cyanotic episodes occurred and he requirec intubation and ventilation. Repeated attempts to wean him off ventilation failed. Hypoxia and hypocapnia repeatedly developed when the ventilator rates were reduced below 15 breaths per minute.

Primary cardiac, pulmonary and neuromuscular abnormalities were excluded. Respiratory monitoring demonstrated hypoventilation during sleep. Respiratory stimulants did not produce a sustained increase in respiratory effort. Congenital central hypoventilation syndrome was diagnosed and a tracheostomy fashioned to facilitate subsequent management.

The clinical course was complicated. A functional low intestinal obstruction presented on the seconc day of life. Radiological appearances were typical of Hirschsprung's disease but rectal biopsies were repeatedly normal. A defunctioning colostomy was performed.

Generalised convulsions occurred at 6 months of age anc treatment with anticonvulsants resulted in loss of awake control of respiration. Now, at the age of 10 months, he requires ventilator support whilst awake and asleep. His cevelopment is difficult to assess but is delayed by approximately 3 months. We hope eventually to establish him on home ventilation with phrenic nerve pacing a possible option for the future.

Royal Hospital for Sick Children, Edinburgh.

INTRAUTERINE INTRAVENTRICULAR HAEMORRHAGE

H. F. Stirling, J. K. Brown

A previously well 23-year-old was admitted at thirty f'ive weeks with diarrhoea and vomiting. The foetal biophysical prof'ile was abnormal and uItrasound scan revealed a haemorrhage into the right choroid plexus. The illness settled and repeat biophysical profile was normal. Repeat scaming of the foetal head at thirty six weeks confirmed the haemorrhage and showed progressive post-haemorrhagic hydrocephalus. The infant was electively delivered, and was in good condition with no dysmorphic features. His weight was on the 10th centile, his occipitofrontal circumference on the 97 th centile. He was irritable with extensor hypertonus.

Post-natal ultrasound scanning confirmed haemorrhage from the choroid plexus extending into the lateral ventricies and midine structures. Doppler studies of cerebral blood flow suggested the intracranial pressure was raised, which was confirmed by ventricular puncture. When the pressure was controlled, using a Rickham reservoir followed by Puäenz-Schulk ventriculoperitoneal. shunt, normal cerebral blood plow was restored.

Sequential ultrasound scans confirmed resolution of the haemorrhage and return of the ventricular system to normal. The infant thrived and has no focal neurological deficits at 3 months of age.

The spectrum of antenatal intracranial haemorrhage is discussed. The significance of raised intracranial pressure and its effects on cerebral blood flow in the neonate is demonstrated. The value of ultrasound and related techniques was stressed in both the antenatal and post-natal management of this unusual condition.

Royal Hospital for Sick Children, Edinburgh.

FANLILIAL PERIODIC PARALYSIS OF THE HYPERKALAEMIC TYPE W. B. Wallace, G. Stark

Christopher is a 14-year-old boy with an 11 year history of almost daily attacks of intermittent weakness and stiffness involving mainly his limbs, lasting 1-2 hours, usually precipitated by rest following heavy exercise, not related to cold and may be relieved by exercising the affected parts.

His mother was similarly affected, the disease having now burnt itself out.

Examination findings incluce a muscular physique, mild calf hypertrophy, no evi.dence of myotonia and normal. neurological examination.

Investigations revealed normal resting serum potassium and electromyography. A potassium provocation test demonstrated increased serum potassium, stiffness of 
limbs, inability to stand from lying, abolished tendon reflexes and weaness, lasting 2 hours.

Treatment with a Thiazide diuretic has relieved the symptoms.

The association of episodic muscular paralysis with extracellular potassium shifts presents a challenge to the neurophysiologist. Excitation-contraction coupling and the contractile system function normally and the defect is believed to be due to muscle membrane inexcitability due to altered permiability of the membrane to sodium and/or potassium.

Our presentation of this rare condition will help to increase the awareness of clinicians to a symptom complex which may be too readily dismissed and yet is amenable to simple, safe treatment.

Royal Hospital for Sick Children, Ecinburgh.

\section{Scientific communications}

PULMONARY VALVE STENOSIS: AN UPDATE

M. K. Lim, K. M. Tan, A. B. Houston, W. B. Doig,

E. N. Coleman

Recent developments in both the assessment and the treatment of pulmonary valve stenosis have transformed patient management, as exemplified by the findings in 28 consecutive infants and children with pulmonary valve stenosis undergoing catheterisation with a view to valvuloplasty.

Comparison of Doppler assessment of valve gradient wi.th that measured simultaneously at catheterisation before and after valuuloplasty ( 45 studies) showed an excellent correlation ( $r=0.91$ ) but initial out-patient studies (child not sedated) showed this value to be significantly above that obtained with the patient sedated at catheterisation.

Valvuloplasty was attempted in 22: a clinically important reduction in the gradient occurred in 19 (1 subsequently rising and requiring further valvuloplasty); no significant change occurred in 2; the valvuloplasty catheter could not be passed into the pulmonary artery in ane infant.

However Doppler study $>48$ hours after the procedure demonstrated a clinically significant change in 2 patients; 1 rose from below to above clinically significant levels (requiring further valvuloplasty) and 1 fell from a very high to a low level due to dynamic obstruction, clearly cemonstrated by Doppler which accurately predicted the final gradient.

Thus the assessment of the severity of pulmonary valve stenosis is now best performed non-invasively (both before and after valvuloplasty) with Doppler ultrasound and catheterisation has nothing to add to this. Catheterisation is undertaken for effective management by balloon valvuloplasty, surgery being required infrequently. This obviates unnecessary catheterisation and greatly reduces the need for expensive and potentially dangerous surgery.

Royal Hospital for Sick Children, Yorkhill, Glasgow.

PERINATAL VARICELLA EXPOSURE - THE ABERDEEN EXPERIENCE P. E. Carter, F. Duffty, D. J. Lloyd

Chickenpox is a minor disease of childhood but to the immuno compromised and to the neonate, it may be fatal. As deaths have been reported despite prophylactic immunoglobulin, we now use acyclovir additionally in all. exposed infants where the mother is not known to be immune. we report our experience with fifteen infants over the last two years.

Nine were born to infected mothers and six were in contact with affected siblings. All received immunoglobulin, two were given prophylactic oral acyclovir and eight intravenous acyclovir. Two mothers were treated antenatally with acyclovir and in one, labour was deliberately postponed for one week. Mild chickenpox developed in five infants, of whom four were born to infected mothers. There were no deaths. Three infants receiving acyclovir developed mild skin desquamation and three transient feeding problems.
We recommend that where possible infected mothers should be treated before delivery. In the absence of known maternal immunity, all infants exposed to chickenpox should receive both adequate doses of immunoglobulin and seven days of intravenous acyclovir.

Department of Child Health, University of Aberdeen, Aberceen.

MEDIUM CHAIN ACYL-COA DEHYDROGENASE DEFICIENCY MASQUERADING AS REYE'S SYNDROME: A STUDY OF THE SURVIVORS OF REYE'S SYNDROME TO DETERMINE THE FREQUENCY OF MCAD DEFICIENCY IN THESE PATIENTS

C. D. C. Rittey, J.B. P. Stephenson

Seven children who had Communicable Diseases Surveillance Unit criteria for Reye's syndrome (Unexplained encephalopathy plus EITHER liver histology with characteristic fatty change OR serum transaminases raised $>100$ I.U./1 without hyperbilipubinaemia) and dicarboxylic aciduria during the acute illness were given a single dose of L-cannitine and Phenylpropionic acid on separate days. Urine was collected for a period of six hours following each cose. Five of the children showed normal metabolism of the substances, one showed unequivocal evidence of medium chain acyl-CoA dehydrogenase (MCAD) deficiency and in one child the possibility of ketothiolase deficiency is being further evaluated.

While the clinical and biochemical features of the child with MCAD deficiency fulfilled the CDSU criteria for Reye's syndrome the mode of presentation was atypical. The origin of the encephalopathy was considered to be metabolic and the investigation of the child during the acute illness was directed to that end.

Royal Hospital for Sick Children, Yorkhill, Glasgow.

HOWE VISITING FOR INFANTS DISCHARGED FROM NEONATAL INTENSIVE CARE UNIT

L. AlRoomi, Miss M Goodall, P. Galea

An increasing number of infants who required a prolonged period of neonatal intensive and special care are now surviving. A system of support from trained neonatal staff during the initial period at home seems prudent.

Over a period of 17 months (Sept. 1984 - Jan. 1986), 133 such infants were visited with a total of 394 visits (range $1-7$ and mean 3 visits/infant). The duration of visiting varied from one day to 22 days with a mean of $7.8 \mp 4.3$ days.

The reasons for visiting were: assisted ventilation in $51(38 \%)$, birth weight $\leqslant 1500 \mathrm{~g}$ in $41(30 \%)$, bronchopulmonary dysplasia in $8(6 \%)$, major congenital malformation in $10(7.5 \%)$, drug or alcohol abuse in the parents in $5(3.7 \%)$. At the time of discharge 51 infants (38\%) weighed $\leqslant 2200 \mathrm{~g}$. Infants thrived better at home despite poor home conditions. The mean dajly weight gain before and after discharge/infant was $27.3 \mathrm{~g}$ and $34.4 \mathrm{~g}$.

Problems encountered during visits and dealt with directly were 64 in 51 infants. However 15 other infants were referred back to paediatricians and 7 of them required hospital admission.

117 questionnaires were sent to parents later. All who replied (72) found the scheme helpful particularly for support and reassurance with many encouraging remarks.

Glasgow Royal Maternity Hospital, Rottenrow, Glasgow.

The Seventh Glaxo Scottish Paediatric Society Jubilee Lecture.

Prevention and Prenatal Diagnosis of Congenital Diseases Professor David Brock, Department of Human Genetics, Western General Hospital, Edinburgh.

To be published elsewhere. 